\title{
Recent Results on Muon Capture for a Neutrino Factory and Muon Collider
}

\author{
David Neuffer
}

Fermilab

PO Box 500, Batavia IL 60510

\begin{abstract}
Scenarios for capture, bunching and phase-energy rotation of $\mu$ 's from a proton source have been developed. The goal is capture of a maximal number of muons in a string of $\mathrm{rf}$ bunches with applications in neutrino factories and $\mu^{+}-\mu^{-}$colliders. In this note we begin with the bunching, phase rotation and cooling scenario used in neutrino factory study $2 \mathrm{~B}$ and adapted by $R$. Palmer as the initial stage of a $\mu^{+}-\mu^{-}$collider scenario. However the scenario produces a relatively large number of bunches that must be recombined for maximal collider luminosity. In this paper we modify the scenario to obtain a smaller number of bunches, and, after some optimization, obtain cases that are better for both $v$-factory and collider scenarios. We describe these examples and consider some variations toward an optimal $v$-factory + collider scenario.
\end{abstract}

\section{Introduction}

For a neutrino factory or a $\mu^{+}-\mu^{-}$collider, short, intense bunches of protons are focused onto a target to produce pions, that decay into muons, which are then cooled and accelerated into a high-energy storage ring, where $\mu$ decays can provide beams of high-energy neutrinos for a $v$-factory $[1,2,3]$. If the $\mu^{+}$and $\mu^{-}$bunches are counter-rotating and focused to collide in an interaction region, high-luminosity $\mu^{+}-\mu^{-}$collisions are possible.[4] A clear difference between neutrino factory and collider requirements is that the collider requires concentrating muons into a small number of highly cooled bunches, while the factory simply requires muon capture. In both cases, the challenge is to collect and accelerate as many muons as possible. The initial proton bunch is relatively short $(\sim 1 \mathrm{~m})$, so the pions (and resulting muons) are initially produced within a short bunch length, but they are produced nonresonantly with a broad energy spread, much larger than the acceptance of the following accelerators. In this paper we discuss and optimize a method for capturing the muons and reducing that energy spread using high frequency rf, while bunching the beam suitably for cooling and acceleration.[5]

In this method, the muons first drift from the production target, lengthening into a long bunch with a high-energy "head" and a low-energy "tail". (see Figs. 1 and 2) Then, the beam is transported though an "adiabatic buncher", a section of rf cavities that gradually increase in gradient and decrease in frequency (from $\sim 300$ to $\sim 200 \mathrm{MHz}$, in our initial example). The rf frequency in the buncher is set by requiring that reference particles at fixed energies remain separated by an integer number of wavelengths. This forms the beam into a string of bunches of differing energies. Following the buncher, the beam is transported through an "rf rotator" section that performs a phase-energy rotation that aligns the bunches to (nearly) equal central energies, suitable for injection into a fixed-frequency $\sim 200 \mathrm{MHz}$ cooling system. 
The method was initially developed to obtain muon beams suitable for a neutrino factory and was used in the Study 2B neutrino factory design study.[3] R. Palmer et al. recently noted that the bunches could be recombined after cooling for use in a muon collider. [6] In the present note we consider further optimizations of the method, with a goal of producing higherdensity bunching that is suitable for both $\nu$-factory and $\mu^{+}-\mu^{-}$collider scenarios.

\section{Example: Capture into 201.25 MHz For A Neutrino Factory}

To illustrate the method and its components, we discuss its application to a reference problem of forming a muon bunch with large energy spread into a long string of bunches matched into $\sim 200 \mathrm{MHz}$ rf, and we present simulations of the process, tracking the phaseenergy motion. The example is presented in terms of the parameters used to develop the ICOOL simulation of the buncher-rotator.

The initial beam for the example is obtained from targeting a multi-GeV proton beam bunch onto a heavy metal target immersed in a $20 \mathrm{~T}$ solenoid magnet. (Reference values are 24 $\mathrm{GeV}$ protons, a $\mathrm{Hg}$ target, and $\sim 3 \mathrm{~ns}$ rms bunch length ( $\sim 15 \mathrm{~ns}$ full-width) and the initial $\pi$ distributions were generated using the MARS particle production simulation code. [7]) The magnetic field is adiabatically reduced from $\sim 20 \mathrm{~T}$ to $\sim 2 \mathrm{~T}$ over a distance of $\sim 10 \mathrm{~m}$. This arrangement obtains a secondary pion beam with a broad energy spread, with a large number of low-energy pions ( $100 \mathrm{MeV}$ to $300 \mathrm{MeV}$ kinetic energy). The initial proton bunch is relatively short, and as the secondary pions drift from the target they spread apart longitudinally, developing a position-energy correlation, while decaying into muons. The goal of the buncher/rotator is to capture the maximal number of these low-energy muons into 200 $\mathrm{MHz}$ rf buckets for subsequent cooling and acceleration.

\section{Drift + Adiabatic Buncher}

The target region is followed by an rf-free drift region with length $L_{D}$ where the $\pi$-beam decays, following $(\pi \rightarrow \mu+v)$, and the resulting $\mu$ 's spread out in the longitudinal coordinate $\tau$, following $c \tau(\mathrm{s})=\mathrm{s} / \beta_{\mathrm{z}}+\mathrm{c} \tau_{0}$, where $\mathrm{s}$ is distance along the transport, and $\beta_{\mathrm{z}}=\mathrm{v}_{\mathrm{z}} / \mathrm{c}$ is the longitudinal velocity, with a $c \tau$-E correlation from $1 / \beta_{z}(E)$. The drift is followed by a buncher section with high-frequency rf that forms the beam into a train of bunches.

To obtain buncher parameters, we choose reference particles $(1,2)$ at $\mathrm{P}_{1}=280 \mathrm{MeV} / \mathrm{c}$ and $\mathrm{P}_{2}=$ $154 \mathrm{MeV} / \mathrm{c}$, with the intent of capturing muons in the corresponding energy range ( $\sim 80$ to $\sim 200 \mathrm{MeV})$. The $\mathrm{rf}$ frequency $\mathrm{f}_{\mathrm{rf}}$ and phase are set to place these particles at the center of bunches while the rf voltage increases along the transport and the $\mu$-beam is formed into a string of bunches. These conditions can be maintained if the rf wavelength $\lambda_{\mathrm{rf}}$ increases along the buncher, following:

$$
\mathrm{N}_{\mathrm{B}} \lambda_{\mathrm{rf}}(\mathrm{s})=\mathrm{N}_{\mathrm{B}} \frac{\mathrm{c}}{\mathrm{f}_{\mathrm{rf}}(\mathrm{s})}=\mathrm{s}\left(\frac{1}{\beta_{2}}-\frac{1}{\beta_{1}}\right)
$$

Here $\mathrm{s}$ is the total distance from the target, $\beta_{2}, \beta_{1}$ are the velocities of the reference particle and $\mathrm{N}_{\mathrm{B}}$ is an integer. For Study $2 \mathrm{~B}, \mathrm{~N}_{\mathrm{B}}$ was chosen to be $18, \mathrm{~L}_{\mathrm{D}}$ was $107.2 \mathrm{~m}$ and the total buncher length was $51 \mathrm{~m}$. At these parameters, the rf cavities decrease in frequency from $350 \mathrm{MHz}\left(\lambda_{\mathrm{rf}}=0.857 \mathrm{~m}\right)$ to $237 \mathrm{MHz}\left(\lambda_{\mathrm{rf}}=1.265 \mathrm{~m}\right)$ over the buncher. The rf cavities were $0.5 \mathrm{~m}$ long and placed within $0.75 \mathrm{~m}$ long cells. The rf gradient is increased from cell to cell 
along the buncher, and the beam is "adiabatically" captured into a string of bunches, each of them centered about test particle positions with energies determined by the $\delta(1 / \beta)$ spacing from the initial test particle: $1 / \beta_{\mathrm{i}}=1 / \beta_{1}+\mathrm{n} \delta(1 / \beta)$, where $\delta(1 / \beta)=\left(1 / \beta_{2}-1 / \beta_{1}\right) / \mathrm{N}_{\mathrm{B}}$.

For the baseline case for Study 2B, the cavity gradients follow a linear + quadratic increase:

$$
\mathrm{V}_{\mathrm{rf}}^{\prime}(\mathrm{z})=3\left(\frac{\mathrm{z}}{\mathrm{L}_{\mathrm{B}}}\right)+9\left(\frac{\mathrm{z}}{\mathrm{L}_{\mathrm{B}}}\right)^{2} \mathrm{MV} / \mathrm{m}
$$

where $\mathrm{z}$ is distance along the buncher. The gradient at the end of the buncher is $12 \mathrm{MV} / \mathrm{m}$.

Fig. 2 shows simulation results of an initial example, where the simulations were performed using the ICOOL program.[8] Fig. 2A shows beam as produced at the target, Fig. $2 \mathrm{~B}$ shows beam after the end of the drift, and Fig. $2 \mathrm{C}$ shows the beam at the end of the buncher. The beam is formed into a train of different energy bunches.

\section{Phase-Energy Rotator}

After the muons are sorted into well-defined bunches, the rf system is modified to rotate these bunches to nearly identical energies with the design goal of placing all bunches at the same energy, with the bunch spacing at $\sim 201.25 \mathrm{MHz}$ at the end of the Rotator. Somewhat varying scenarios have been developed, we present the algorithm used in developing the Study 2A baseline.

The rf bunch spacing between reference particles is shifted away from the integer by a factor, labeled $\delta \mathrm{N}_{\mathrm{B}}$. and phased so that the high-energy reference particle would be decelerated and the low-energy one would be accelerated. For the Study $2 \mathrm{~B}$ baseline case, $\delta \mathrm{N}$ $=0.05$ was chosen and the bunch spacing between reference particles was therefore $N_{R}=$ 18.05. The reference particles are decelerated and accelerated at a uniform rate until the end of the rotator region where their energies become approximately equal, with the $N_{R}$ rf wavelength spacing between them maintained by setting the rf parameters. At the end of the rf rotator the rf frequency becomes fixed (at $201.25 \mathrm{MHz}$ ) for subsequent rf cavities as the beam enters an ionization cooling channel.

For the Study 2B example the total length of the rotator region rf frequency is $54 \mathrm{~m}$ and $\mathrm{rf}$ gradient is $12 \mathrm{MV} / \mathrm{m}$ in the $\mathrm{rf}$ cavities ( $\mathrm{rf}$ is in $0.5 \mathrm{~m}$ cavities inside $0.75 \mathrm{~m}$ long cells.) The $\mathrm{rf}$ frequency decreases from 232 to $202 \mathrm{MHz}$ from cavity to cavity over the length of the rotator region.

\section{Cooling Channel}

The Study 2B cooling channel consists of a sequence of identical cooling $1.5 \mathrm{~m}$ cells, as shown in figure 3.[3] Each cell contains $20.5 \mathrm{~m}$-long rf cavities, with $0.25 \mathrm{~m}$ spacing between the cavities. $1 \mathrm{~cm}$ long $\mathrm{LiH}$ blocks are placed at the ends of each cavity (4 per cell); these blocks provide the energy loss for ionization cooling. Each cell contains two solenoidal coils of alternating sign; this provides an approximately sinusoidal variation of the magnetic field in the channel with a peak value of $\sim 2.5 \mathrm{~T}$, providing transverse focusing with $\beta_{\perp} \cong 0.7 \mathrm{~m}$. The currents in the first two cells are perturbed from the reference values to provide matching from the constant-field solenoid in the buncher and rotator section. The total baseline length of the cooling section was $76.5 \mathrm{~m}$ (51 cells). 
The cooling channel cools the rms transverse normalized emittance from $\varepsilon_{\mathrm{N}, \mathrm{rms}}=0.0182$ $\mathrm{m}$. to $\varepsilon_{\mathrm{N}, \mathrm{rms}}=0.00762 \mathrm{~m}$ in simulation. In the Study 2B discussion the cooling was also measured by counting the number of simulation particles that fall within a reference acceptance, which approximated the expected acceptance of the downstream accelerator. For Study $2 \mathrm{~B}$, particles with transverse amplitudes $\mathrm{A}_{\mathrm{T}}$ less than $0.03 \mathrm{~m}$ and longitudinal amplitudes $\mathrm{A}_{\mathrm{L}}$ less than $0.15 \mathrm{~m}$ were considered accepted.

The amplitude $\mathrm{A}_{\mathrm{T}}$ was evaluated by:

$$
A_{T}=\beta \gamma\left[\beta_{\perp}\left(x^{\prime 2}+y^{\prime 2}\right)+\gamma_{\perp}\left(x^{2}+y^{2}\right)+2 \alpha_{\perp}\left(x x^{\prime}+y^{\prime}\right)+2\left(\beta_{\perp} \kappa-L\right)\left(x y^{\prime}-y^{\prime}\right)\right]
$$

where $\beta_{\perp}, \gamma_{\perp}, \alpha_{\perp}$ are betatron functions, and the last term is an angular momentum correction.[9] For longitudinal motion the variables $t_{c}=c \tau$ (phase lag in periods within a bunch multiplied by rf wavelength) and $\delta \mathrm{E}$ (energy difference from centroid) are used (rather

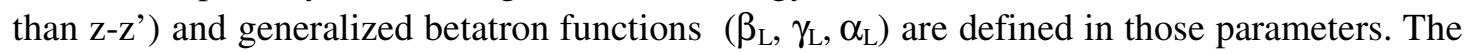
longitudinal amplitude is evaluated by:

$$
A_{L}=\frac{1}{\left(m_{\mu} c^{2}\right)}\left[\gamma_{L} t_{c}^{2}+\beta_{L} \delta E^{2}+2 \alpha_{L} t_{c} \delta E\right] .
$$

(Our notation differs from that in ref. 9, although the same criteria were used.)

Under these criteria, the present example obtained $\sim 0.243 \mu / \mathrm{p}$ in simulation. This was an increase from $\sim 0.117 \mu / \mathrm{p}$ at the beginning of the muon cooling channel; the channel doubled the number of muons within the acceptance. The longitudinal rms emittance was $\sim 0.071 \mathrm{~m}$ at the end of the cooling channel, an increase from $\sim 0.053$ at the beginning; the cooling channel does not have longitudinal cooling.

For study 2, a more restricted criterion of transverse amplitude of $0.015 \mathrm{~m}$ was used; the cooling channel increases beam within that acceptance from $0.044 \mu / \mathrm{p}$ to $0.127 \mu / \mathrm{p}$. For reference comparisons with the studies described below, we also consider increasing the longitudinal acceptance from $0.15 \mathrm{~m}$ to 0.2 and $0.25 \mathrm{~m}$; that increases the Study $2 \mathrm{~B}$ acceptance to $0.265 \mu / \mathrm{p}$ and $0.272 \mu / \mathrm{p}$, respectively.

For the published version of Study 2B,[3] this case was modified toward a more realistic implementation with a reduced set of $\mathrm{rf}$ frequencies, more detailed magnetic and $\mathrm{rf}$ field models, inclusion of rf windows, a differing initial production model, and an older scattering model was used (with larger scattering effects). The published version obtained $\sim 0.17 \mu / \mathrm{p}$ in simulation. We are using the more idealized settings and newer cooling model to obtain more direct comparisons with the following simulations that are similarly calculated.

\section{Comments on example}

This initial example contains many system parameters, and these parameters can be greatly varied in further optimizations. We list some of these key parameters:

0. Proton Source/target: The baseline proton energy $E_{P}$, bunch length $\left(l_{P}\right)$ and intensity $\left(N_{P}\right)$, as well as the target material and length.

1. Drift: The key parameter is the length of the section, $\mathrm{L}_{\mathrm{D}}$. 
2. Buncher: The length of the section $\left(\mathrm{L}_{\mathrm{B}}\right)$, the bunching gradient $\mathrm{E}_{\mathrm{rf}, \mathrm{B}}(\mathrm{z})$, the reference particle momenta $\mathrm{P}_{1}, \mathrm{P}_{2}$ and the reference bunch spacing $\mathrm{n}_{\mathrm{B}}$ can be varied.

3. $\phi-\delta E$ Rotation: The length and rf voltage of the phase rotation section $\left(\mathrm{L}_{\mathrm{R}}\right.$ and $\left.\mathrm{E}_{\mathrm{rf}, \mathrm{R}}\right)$ are the key parameters. Also the reference particle momenta $\left(\mathrm{P}_{1}, \mathrm{P}_{2}\right)$, their rates of change and the vernier parameter $\delta \mathrm{N}_{\mathrm{B}}$ can be changed.

4. Cooling System: The key parameters of the cooling system are the system length $\mathrm{L}_{\mathrm{C}}$, the rf frequency $\mathrm{f}_{\mathrm{rf}}$, the $\mathrm{rf}$ gradient $\mathrm{E}_{\mathrm{rf}, \mathrm{cool}}$, and the absorber material and thickness (for energy loss rate), and the transverse focusing $\left(\beta^{*}(\mathrm{z})\right)$ which determines the transverse cooling limits. All of these parameters can be varied to improve performance.

In the initial example we have separated the adiabatic buncher, the $\phi-\delta \mathrm{E}$ rotation and the cooling section into separated consecutive systems. It is also possible to combine these into a more integrated system.

While the $\mu / \mathrm{p}$ numbers quoted here consider only one sign of $\mu$ 's, the multibunch capture system captures both signs with approximately equal intensities. The initial target produces both $\mu^{+}$and $\mu^{-}$in nearly equal amounts, and, half-way between each of the stable phases for one sign of $\mu$ 's in the buncher and $\phi-\delta \mathrm{E}$ rotator, there is a stable phase for the opposite sign. The same system obtains strings of $\mu$-bunches of both signs.

\section{Extension to $\mu^{+}-\mu^{-}$Collider Scenarios}

The study 2B example obtains a long train of bunches ( $\sim 50+$ bunches, $80 \mathrm{~m}$ long train). As long as the bunch train length is much less than the $\mu$-storage ring circumference, the long train is acceptable for a neutrino factory; the critical quantity is the total number of $\mu$ 's. The system produces bunches of both $\mu^{+}$s and $\mu^{-}$s, and both signs are needed for a $\mu^{+}-\mu^{-}$collider. However, for a collider, high luminosity requires capturing the muons into a small number of bunches, and the long trains are not compatible with this requirement.

R. Palmer et al. recently noted that, in study $2 \mathrm{~B}$, most of the muons are in the first $\sim 21$ captured bunches ( $2 / 3$ of the total), and devised a scenario to cool the muons and recombine these 21 into single bunches, which would then be compatible with a muon collider. [6] About half the muons are lost through decay and mismatch in this process, and it could be improved with a shorter bunch train and smaller number of bunches.

\section{Variations: Shorter bunch trains}

To obtain scenarios more matched to a collider, and to explore further optimizations, we consider modifying the scenario to obtain shorter bunch trains. In the present case we keep the same reference particles, with the goal of capturing similar low-energy muons, and retain the original goal of $\sim 200 \mathrm{MHz}$ bunches, but reduce the reference bunch spacing between them. This necessarily shortens the buncher-rotator system. We consider reducing $\mathrm{n}_{\mathrm{B}}$ from 18 to 10 and also 7 , reducing the system lengths proportionately. We increase rf gradients somewhat from the baseline but with the constraint that the rf gradient in the cavities remain less than $\sim 15 \mathrm{MV} / \mathrm{m}$. The total $\mathrm{rf}$ voltages in the shorter systems are still somewhat less than in the Study $2 \mathrm{~B}$ case. 


$$
N_{B}=10 \text { example }
$$

After some parameter variation we find systems with parameters of the type shown in Table 1. For the $\mathrm{N}_{B}=10$ case the drift + buncher + rotator length was $123.9 \mathrm{~m}$, almost $100 \mathrm{~m}$ shorter than the $N_{B}=18$ case. Figure 4 shows the resulting layout, which can be compared with the longer study 2B case shown in Fig. 1.

The buncher was only $31.5 \mathrm{~m}$ in length, and the rf gradient along the buncher was somewhat increased:

$$
\mathrm{V}_{\mathrm{rf}}^{\prime}(\mathrm{z})=6\left(\frac{\mathrm{z}}{\mathrm{L}_{\mathrm{B}}}\right)+9\left(\frac{\mathrm{z}}{\mathrm{L}_{\mathrm{B}}}\right)^{2} \mathrm{MV} / \mathrm{m}
$$

The rf frequency decreased from 360 to $240 \mathrm{MHz}$ along the buncher.

The rotator is the same as that used in study $2 \mathrm{~B}$, except that it was much shorter $(54 \mathrm{~m} \rightarrow$ $36 \mathrm{~m}$ ), and the maximum rf gradient within the cavities was increased from 12 to $15 \mathrm{MV} / \mathrm{m}$. The total rf voltage is reduced from 432 to $360 \mathrm{MV}$.

After cooling to $\varepsilon_{\mathrm{t}}=0.0078 \mathrm{~m}$ in $\sim 80 \mathrm{~m}$, the acceptance within the Study $2 \mathrm{~B}$ baseline $\left(\mathrm{A}_{\mathrm{T}}<\right.$ $0.03 \mathrm{~m} \mathrm{~A}_{\mathrm{L}}<0.15 \mathrm{~m}$ ) was $\sim 0.237 \mu / \mathrm{p}$, increased from 0.109 at the end of the rotator. The longitudinal emittance per bunch was slightly greater than in the $\mathrm{N}_{\mathrm{B}}=18$ case, $\varepsilon_{\mathrm{L}, \mathrm{Nrms}}=$ $0.078 \mathrm{~m}$. With the larger emittance, it is more desirable to increase the longitudinal acceptance. An increase to 20 or $25 \mathrm{~cm}$ increased the $\mu / \mathrm{p}$ to 0.262 and 0.28 , respectively.

Simulation results are shown in fig. 5. This shorter system produced about the same number of muons as the longer $\mathrm{N}_{\mathrm{B}}=18$ case, but captured them into a much shorter bunch train (by 10/18). 12 of these bunches contain as many muons as $\sim 21$ bunches of the longer bunch train, and these bunches could be much more easily recombined for a collider scenario. The densest bunches in this $\mathrm{N}_{\mathrm{B}}=10$ case had about twice as many muons as the densest bunches in the $\mathrm{N}_{B}=18$ case. Because of the shorter bunch train, the present example is significantly more effective than the Study 2B example.

$$
N_{B}=7 \text { Example }
$$

We considered further reduction of the bunch train length by using $\mathrm{N}_{B}=7$, and the resulting parameters are displayed in Table 1 . The drift + buncher + rotator system length is reduced to $85.75 \mathrm{~m}$, less than half of the Study 2B example.

The buncher is only $21 \mathrm{~m}$ in length, and the rf gradient along the buncher increases linearly along the transport:

$$
\mathrm{V}_{\mathrm{rf}}^{\prime}(\mathrm{z})=15\left(\frac{\mathrm{z}}{\mathrm{L}_{\mathrm{B}}}\right) \mathrm{MV} / \mathrm{m} \text {. }
$$

The rf frequency decreases from 360 to $240 \mathrm{MHz}$ along the buncher.

The rotator $(27 \mathrm{~m})$ is shorter than the previous cases, and the maximum rf gradient within the cavities was $15 \mathrm{MV} / \mathrm{m}$. The total $\mathrm{rf}$ voltage is reduced to $270 \mathrm{MV}$. The $\mathrm{rf}$ frequency decreases from $\sim 240 \mathrm{MHz}$ to $202 \mathrm{MHz}$ along the rotator length. 
After cooling to $\varepsilon_{\mathrm{t}}=0.008 \mathrm{~m}$ in $80 \mathrm{~m}$, the acceptance within the Study $2 \mathrm{~B}$ baseline $\left(\mathrm{A}_{\mathrm{T}}<0.03 \mathrm{~m}\right.$ $\mathrm{A}_{\mathrm{L}}<0.15 \mathrm{~m}$ ) is $\sim 0.181 \mu / \mathrm{p}$, increased from 0.098 at the end of the rotator. The longitudinal emittance per bunch is greater than in the previous cases, $\varepsilon_{\mathrm{L}, \mathrm{Nrms}}=0.091 \mathrm{~m}$. With the larger emittance, it is desirable to increase the longitudinal acceptance. An increase of $A_{L}$ to 20 or $25 \mathrm{~cm}$ increased the $\mu / \mathrm{p}$ to 0.212 and 0.231 , respectively. These numbers are about $20 \%$ less than the previous cases. The most dense bunches have roughly the same number of muons as those of the $\mathrm{N}_{\mathrm{B}}=10$ cases. The bunch train is shorter, and the reduced capture numbers are due to that reduction in bunch train length. The larger rms emittance per bunch obtains a similar total longitudinal emittance.

Thus, this shorter system captures fewer muons than the longer $\mathrm{N}_{B}=10$ case, but it captures them into a shorter bunch train (by $\sim 20 \%$ ). The system is not as effective as the $\mathrm{N}_{\mathrm{B}}=10$ case, but may be less expensive due to the shorter overall length of the system.

\section{Variation: Better Transverse Cooling}

The performance in this and previous examples is clearly limited by the transverse cooling system, which provides a relatively limited amount of cooling. The cooling is limited by the choice of absorber $(\mathrm{LiH})$ and by the relatively weak focusing lattice. Both of these factors limit the possible cooling., as shown in the cooling equation:

$$
\frac{\mathrm{d} \varepsilon_{\mathrm{N}}}{\mathrm{ds}}=-\frac{1}{\beta \mathrm{cp} p_{\mu}} \frac{\mathrm{dE}}{\mathrm{ds}} \varepsilon_{\mathrm{N}}+\frac{\beta \gamma \beta_{\perp}}{2} \frac{\mathrm{E}_{\mathrm{s}}^{2}}{\beta^{2} \mathrm{c}^{2} \mathrm{p}_{\mu}^{2} \mathrm{~L}_{\mathrm{R}}},
$$

where $\mathrm{dE} / \mathrm{ds}$ is the energy loss in material, $\mathrm{E}_{\mathrm{s}}=\sim 14 \mathrm{MeV}, \mathrm{L}_{\mathrm{R}}$ is the material radiation length, $\beta=\mathrm{v} / \mathrm{c}$ and $\gamma$ are the usual kinematic factors and $\beta_{\perp}$ is the betatron function, which is $\cong 0.8 \mathrm{~m}$ in the Study $2 \mathrm{~B}$ lattice. The equilibrium emittance $\left(\sim \beta_{\perp} \mathrm{E}_{\mathrm{s}}{ }^{2} /\left(\beta \mathrm{m}_{\mu} \mathrm{c}^{2} \mathrm{dE} / \mathrm{ds}_{\mathrm{R}}\right)\right)$ is $\varepsilon_{\mathrm{eq}} \sim 0.006 \mathrm{~m}$ with $\mathrm{LiH}$ absorbers in this lattice.

In the present case, we consider replacing the $\mathrm{LiH}$ material by hydrogen, which has an equilibrium emittance that is roughly half of $\mathrm{LiH}\left(\varepsilon_{\mathrm{eq}} \cong 0.003 \mathrm{~m}\right)$. The other cooling parameters (the cell length and focusing magnets), and the rf voltage and phase remain the same. (see fig. 6) (A similar reduction in $\beta_{\perp}$ by a factor of two at the $\mathrm{LiH}$ absorbers should yield a similar improvement in cooling.) To simplify discussion, we use Hydrogen gas distributed throughout the system. This would correspond to using gas-filled rf cavities and transport, which could be desirable to use in this application because the gas would suppress rf breakdown that may otherwise occur in vacuum cavities within the large magnetic focusing fields. Matching the energy loss to the previous scenario obtains a gas density corresponding to $120 \mathrm{Atm}$ at $\operatorname{STP}(0.142$ of liquid density).

The hydrogen gas absorber enables cooling to smaller beam size and superior acceptance. (see fig. 7) With liquid hydrogen absorbers, cooling to $\varepsilon_{\mathrm{N}, \mathrm{rms}}=0.0064 \mathrm{~m}$ with $\sim 80 \mathrm{~m}$ in cooling is obtained and the number of muons within the Study $2 \mathrm{~B}$ acceptance for $\mu / \mathrm{p}$ is increased to $0.286 \mu / \mathrm{p}\left(\right.$ at $\left.\mathrm{N}_{\mathrm{B}}=10\right)$, an increase of $\sim 20 \%$. Extending longitudinal acceptance to 20 and $25 \mathrm{~cm}$ amplitudes increases this to 0.326 and $0.35 \mu / \mathrm{p}$; this is roughly $25 \%$ more than with $\mathrm{LiH}$. The number of muons within the reference acceptances is significantly increased. The improved cooling also increases acceptance into the Study 2 acceptance $\left(\mathrm{A}_{\mathrm{T}}<0.015 \mathrm{~m}\right)$. That acceptance is increased to $0.172,0.20$ and $0.215 \mu / p$ for $A_{L}=0.15,0.20,0.25$, respectively. 
For the $N_{B}=7$ example, similar improvements also occur: $\mu / p$ at $A_{L}<0.2 m$ increases from $\sim 0.21$ to 0.25 , and other cases have an $\sim 20 \%$ improvement.

\section{Comments and Future Studies}

From these initial evaluations, the $\mathrm{N}_{\mathrm{B}}=10$ example seems preferable to the baseline Study 2B. It produces a shorter bunch train within a more compact system, with as many muons for a neutrino factory as the baseline. The $\mathrm{N}_{\mathrm{B}}=10$ system, with somewhat reduced transport and integrated rf requirements, should also be somewhat less expensive. It may therefore now be desirable for it to be developed to the level of realistic design detail used in Study 2B and the ISS $v$-factory study, so that more accurate value and performance calculations can be obtained. The example would also be more useful as the initial beam for a muon collider, since the shorter bunch train is much more easily recombined further downstream.

From the above discussion and simulations, the $\mathrm{N}_{\mathrm{B}}=7$ example is somewhat less effective. While peak bunch densities are similar, the shorter bunch train does not capture as many muons. The shorter system may be more affordable and may have advantages in a collider scenario; however, and other optimizations (possibly with more rf gradient and/or cooling) may make it more attractive.

The baseline cooling system only cools transversely by a factor of $\sim 2.5$ in transverse emittance, and increases the muons accepted into a $v$-factory by a factor of $\sim 2$. As discussed here, a stronger cooling system could improve performance. The cooling could be improved by using a different cooling material $\left(\mathrm{H}_{2}\right)$ or stronger focusing. Also the cooling system does not include any longitudinal cooling, and modification to include longitudinal cooling could be a significant improvement.

The present study only covered a small range of the optimization possibilities and many other options could be explored. We will explore other variations in further studies and invite the reader(s) to suggest other possibilities and to develop these concepts as well.

\section{Acknowledgments}

I thank R. Palmer and A. Van Ginneken for important contributions in the development of this concept. I also acknowledge important contributions from R. Fernow, J. Gallardo, K. Paul and C. Yoshikawa. Research supported by Department of Energy under contract no. DEAC02-07CH11359 and DOE STTR Grant DE-FG02-05ER-86252. 


\section{REFERENCES}

1. N. Holtkamp and D. Finley, eds., "A Feasibility Study of a Neutrino Source based on a Muon Storage Ring", Fermilab-Pub-00/108-E (2000).

2. S. Ozaki, R. Palmer, M. S. Zisman, Editors, "Feasibility Study-II of a Muon-Based Neutrino Source", BNL52623, June 2001. http://www.cap.bnl.gov/mumu/studyII/FS2-report.html.

3. J. S. Berg, S. A. Bogasz, S. Caspi, J. Cobb, R. C. Fernow, J. C. Gallardo, S. Kahn, H. Kirk, D. Neuffer, R. Palmer, K. Paul, H. Witte, M. Zisman, "Cost-effective Design for a Neutrino Factory", Phys. Rev. STAB 9,011001(2006).

4. The $\mu^{-}-\mu^{+}$Collaboration, Report BNL-52503, Fermi Lab-Conf. 96/092, LBNL-38946, presented at Snowmass96, (1996).

5. D. Neuffer and A. Van Ginneken, Proc. 2001 Particle Acc. Conf., Chicago, p. 2029 (2001), D. Neuffer, "'High Frequency' Buncher and Phase Rotation”, Proc. NuFACT03, AIP Conf. Proc. 721, p. 407. (2004).

6. R. Palmer et al. , "A Complete Scheme for a Muon Collider", NFMCC-doc-519, presented at COOL07, Bad Kreuznach, Germany, September 2007.

7. N. Mokhov, "Particle Production and Radiation Environment at a Neutrino Factory Target Station", FermilabConf-01/134, Proc. 2001 Particle Accelerator Conference, Chicago, P. 745 (2001), see http://wwwap.fnal.gov/MARS/

8. R. Fernow, "ICOOL, a Simulation Code for Ionization Cooling of Muon Beams", Proc. 1999 Particle Accelerator Conference, New York, p. 3020 (1999), see http://pubweb.bnl.gov/people/fernow/.

9. R. Fernow, "Physics Analysis Performed by ECALC9", Mucool Note 280, September 2003. 


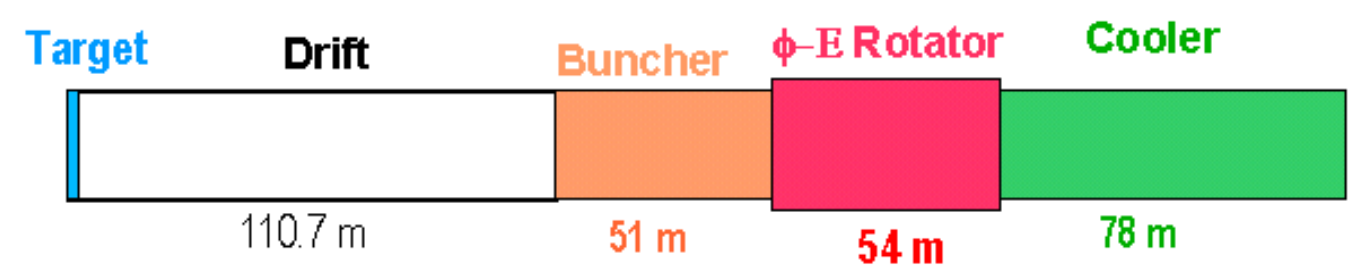

FIGURE 1. Schematic view of the components of the Study 2B front-end transport system, showing an initial drift $(107 \mathrm{~m})$, the varying frequency buncher $(51 \mathrm{~m})$, and the phase-energy $(\phi-\delta \mathrm{E})$ rotator $(54 \mathrm{~m})$ leading into a cooling section of $78 \mathrm{~m}$. $\pi$ 's would be produced by protons on a target at the beginning of the drift, decay to $\mu$ 's in the drift, while lengthening in phase. The buncher and $\phi-\delta \mathrm{E}$ rotator form the $\mu$ 's into a string of bunches matched into the cooler.

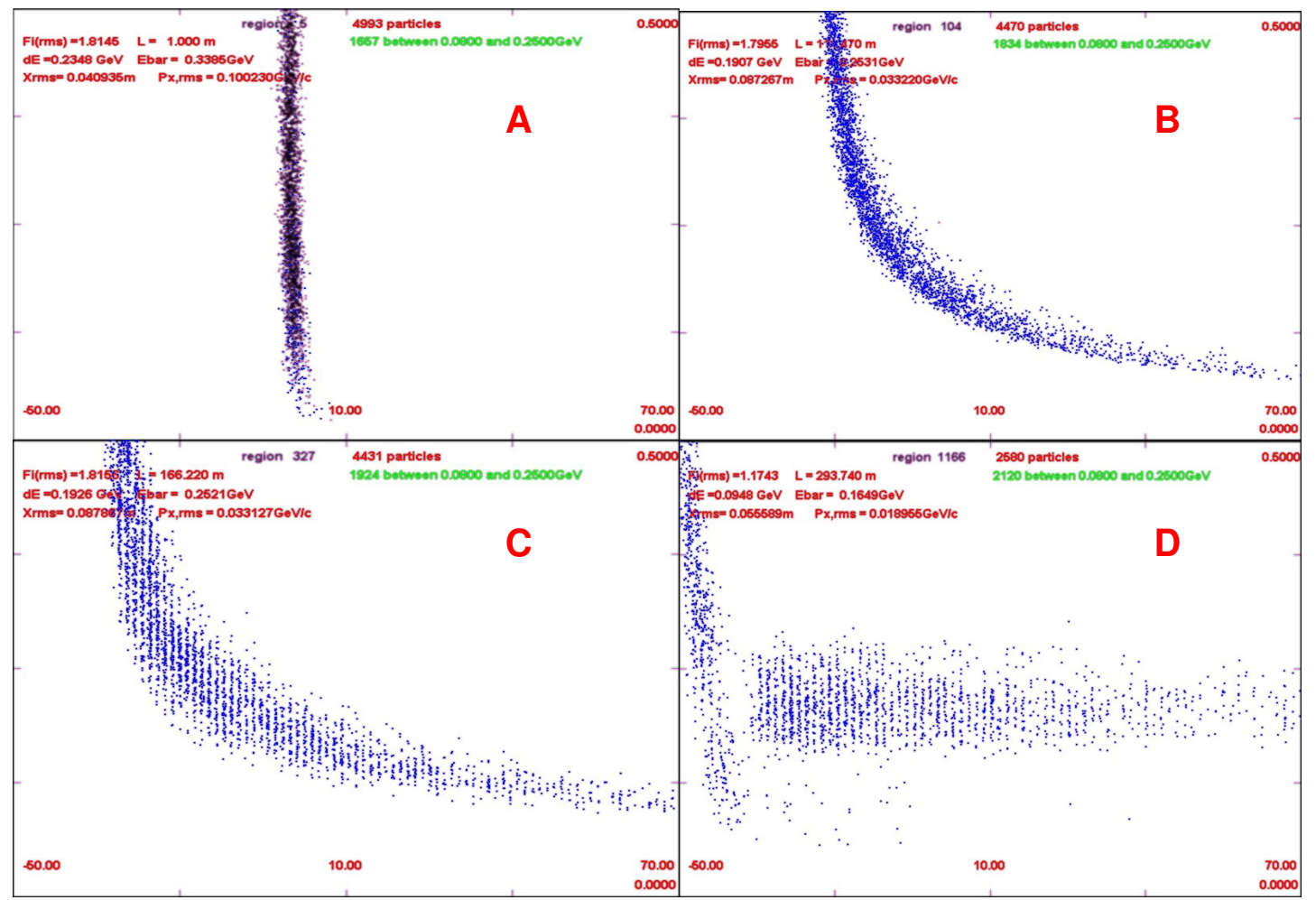

Figure 2: ICOOL simulation results of the buncher and phase rotation, at the parameters of the initial Study2B example described in the text. Each figure shows: A: $\pi$ 's and $\mu$ 's as produced at the end of a $1.0 \mathrm{~m}$ long target. B: $\mu$ 's at $\mathrm{z}=112 \mathrm{~m}$ after a drift . C: $\mu$ 's at $\mathrm{z}=166 \mathrm{~m}$, the end of the buncher. The beam has been formed into a string of $\sim 200 \mathrm{MHz}$ bunches at different energies. D: At $\mathrm{z}=294 \mathrm{~m}$ after $\phi-\delta \mathrm{E}$ rotation and $\sim 80 \mathrm{~m}$ of cooling ; the bunches are aligned into nearly equal energies, and transverse emittance has been reduced by a factor of $\sim 2$. In each plot the vertical axis is momentum ( 0 to 0.5 $\mathrm{GeV} / \mathrm{c}$ ) and the horizontal axis is longitudinal position with respect to a reference particle (-50 to $70 \mathrm{~m})$. 


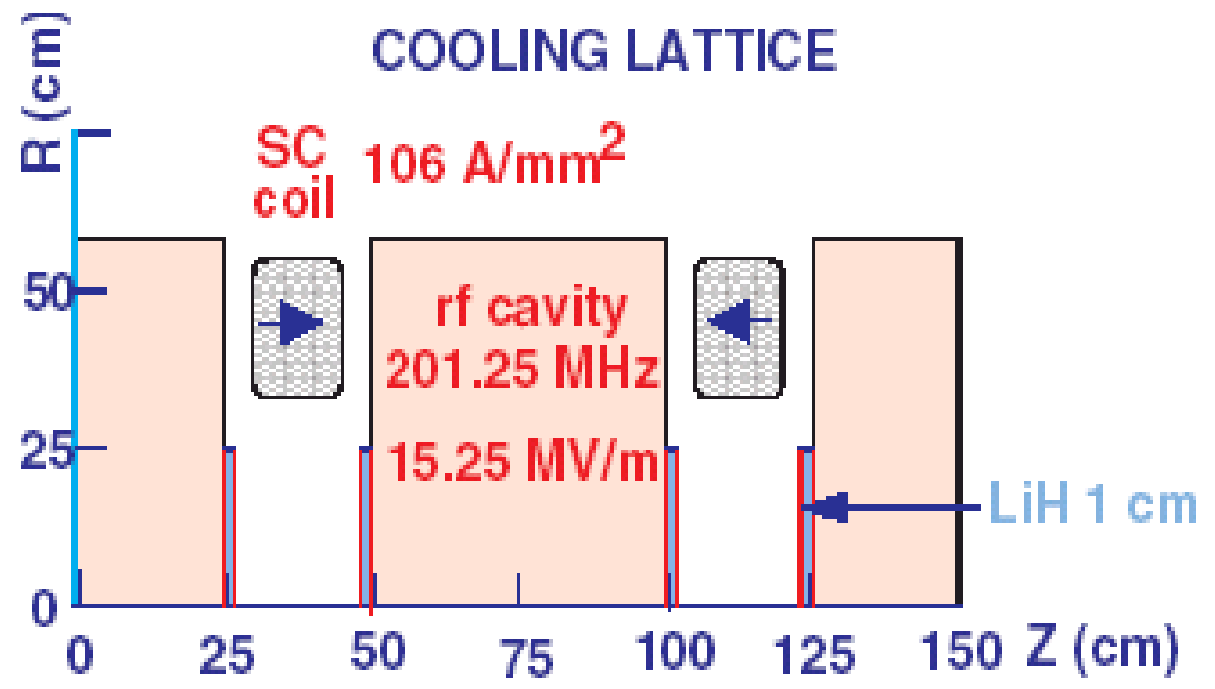

Figure 3: Cooling cell from Study 2B.[3] The cooling cell includes $2 \mathrm{rf}$ cavites , $4 \mathrm{LiH}$ absorbers and two superconducting coils. 
Table 1: Parameters of some buncher/rotator scenarios.

\begin{tabular}{|l|l|l|l|l|}
\hline Simulation cases & & Study 2B & & \\
\hline Parameter & & $\mathrm{N}_{\mathrm{B}}=18$ & $\mathrm{~N}_{\mathrm{B}}=10$ & $\mathrm{~N}_{\mathrm{B}}=7$ \\
\hline Bunch spacing number & $\mathrm{N}_{\mathrm{B}}$ & 18 & 10 & 7 \\
\hline Drift Length & $\mathrm{L}_{\mathrm{D}}$ & $110.7 \mathrm{~m}$ & $56.4 \mathrm{~m}$ & $37.75 \mathrm{~m}$ \\
\hline Buncher Length & $\mathrm{L}_{\mathrm{B}}$ & $51 \mathrm{~m}$ & 31.5 & 21 \\
\hline Buncher rf Gradient & $\mathrm{V}_{\mathrm{rf}}$ & 0 to 12 & 0 to 15 & 0 to 15 \\
\hline Buncher rf frequencies & $\mathrm{f}_{\mathrm{rf}, \mathrm{B}}$ & $360 \rightarrow 235 \mathrm{MHz}$ & $360 \rightarrow 240$ & $350 \rightarrow 240 \mathrm{MHz}$ \\
\hline Rotator Length & $\mathrm{L}_{\mathrm{R}}$ & $54 \mathrm{~m}$ & $36 \mathrm{~m}$ & $27 \mathrm{~m}$ \\
\hline Rotator Bunch spacing & $\mathrm{N}_{\mathrm{B}}+\delta \mathrm{N}_{\mathrm{B}}$ & 18.05 & 10.07 & 7.08 \\
\hline Rotator gradient & $\mathrm{V}_{\mathrm{rf}}$ & 12 & 15 & 15 \\
\hline Rotator rf frequencies & $\mathrm{f}_{\mathrm{rf}, \mathrm{R}}$ & 232 to 202 & 240 to 201.5 & 240 to 201.5 \\
\hline $\begin{array}{l}\mu / \mathrm{p} \quad\left(\mathrm{A}_{\mathrm{T}}<0.03, \mathrm{~A}_{\mathrm{L}}<0.2\right) \\
\text { after rotator. }\end{array}$ & $\mu / \mathrm{p}$ & 0.126 & 0.124 & 0.10 \\
\hline $\begin{array}{l}\left.\mu / \mathrm{p} \quad \mathrm{A}_{\mathrm{T}}<0.03, \mathrm{~A}_{\mathrm{L}}<0.2\right) \\
\text { After LiH cooler }\end{array}$ & $\mu / \mathrm{p}$ & 0.265 & 0.263 & 0.23 \\
\hline $\begin{array}{l}\left.\mu / \mathrm{p} \quad \mathrm{A}_{\mathrm{T}}<0.03, \mathrm{~A}_{\mathrm{L}}<0.2\right) \\
\quad \text { After } \mathrm{H}_{2} \text { cooler }\end{array}$ & $\mu / \mathrm{p}$ & 0.342 & 0.326 & 0.251 \\
\hline Final transverse emittance & $\varepsilon_{\mathrm{T}, \mathrm{rms}}$ & 0.0076 & 0.0078 & 0.008 \\
\hline Final Longitudinal emittance & $\varepsilon_{\mathrm{L}, \mathrm{rms}}$ & 0.071 & 0.076 & 0.091 \\
\hline & & & & \\
\hline
\end{tabular}


Figure 4: Layout of the $\mathrm{N}_{\mathrm{B}}=10$ candidate (Buncher-Rotator-Cooling) system. The total length is $\sim 204 \mathrm{~m}$, and is $\sim 2 / 3$ that of Study $2 \mathrm{~B}$. The drift, buncher and $\phi$-E rotator are shortened.
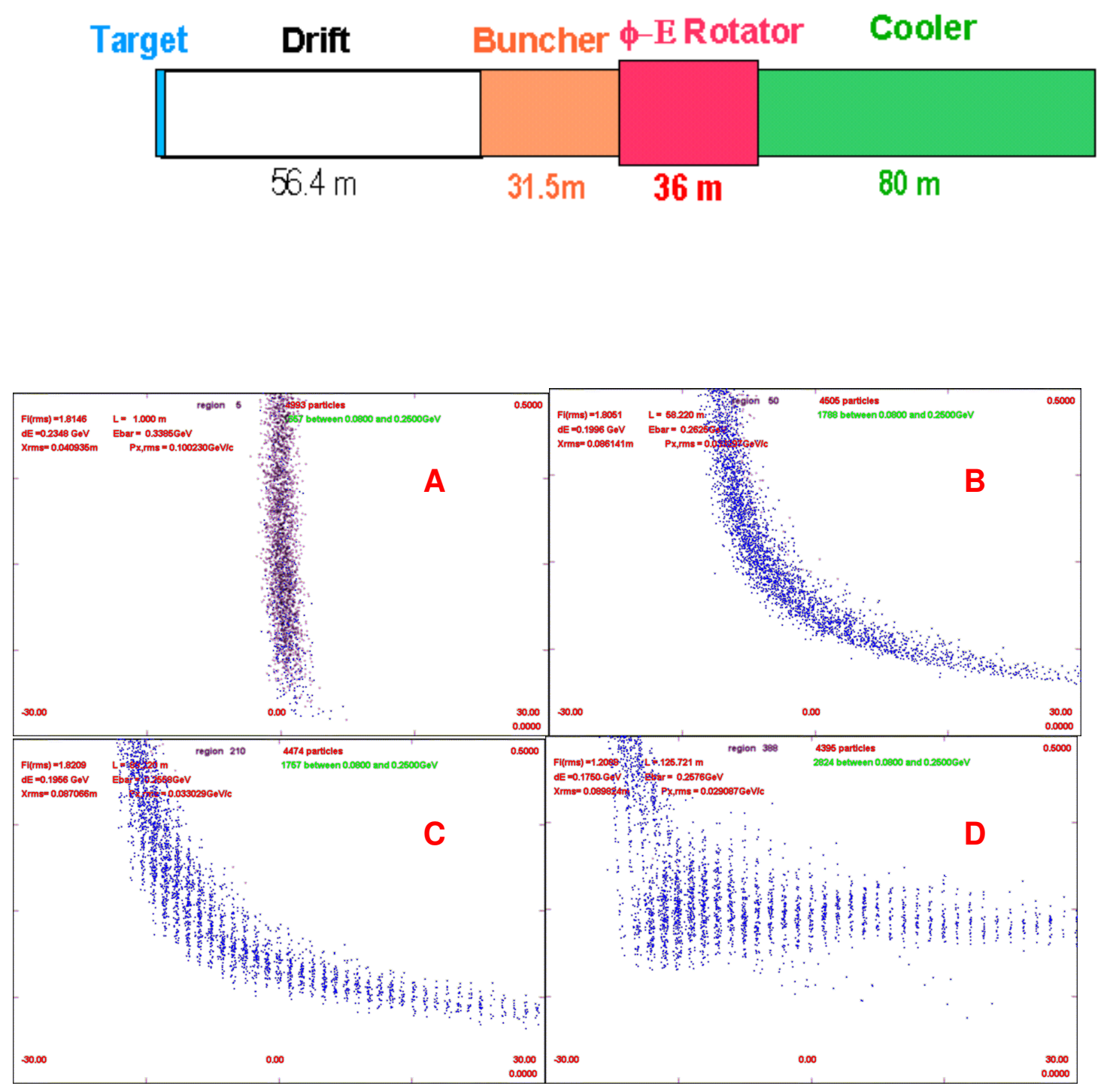

Figure 5: ICOOL simulation results of the buncher and phase rotation, at the parameters of the $\mathrm{N}_{B}=10$ example described in the text. Each figure shows the A: $\pi$ 's and $\mu$ 's as produced at the end of a $1.0 \mathrm{~m}$ long target. B: $\mu$ 's at $z=58 \mathrm{~m}$ after a drift . C: $\mu$ 's at $\mathrm{z}=93 \mathrm{~m}$, the end of the buncher. The beam has been formed into a string of $\sim 200 \mathrm{MHz}$ bunches at different energies. $\mathrm{D}$ : At $\mathrm{z}=126 \mathrm{~m}$ after $\phi-\delta \mathrm{E}$ rotation; the bunches are aligned into nearly equal energies. In each plot the vertical axis is momentum ( 0 to $0.5 \mathrm{GeV} / \mathrm{c}$ ) and the horizontal axis is longitudinal position with respect to a reference particle ( -30 to $30 \mathrm{~m})$. 
Figure 6: Layout of the cooling cell with $\mathrm{H}_{2}$ gas absorber, from center of coil to center of coil

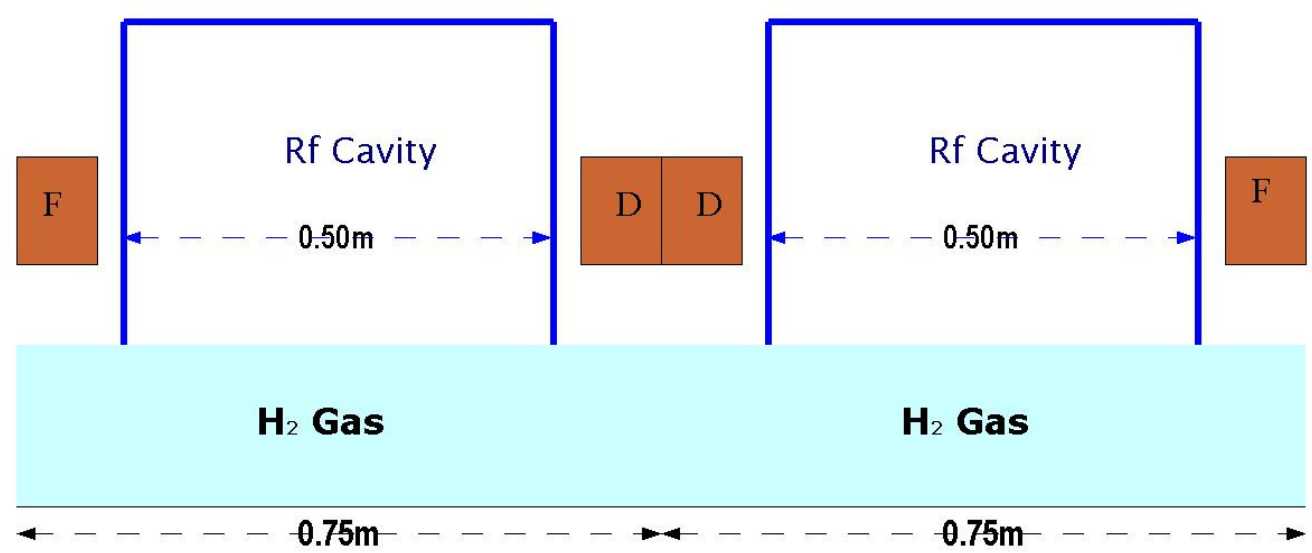

Figure 7: Transverse cooling in the neutrino factory cooling channel. Figures show transverse beam coordinates. (x-y) Horizontal and vertical scales extend from -0.4 to $0.4 \mathrm{~m}$. A: Beam at start of cooling channel. B: beam at end of channel using $\mathrm{LiH}$ absorbers. $\mathrm{C}$ : Beam at end of channel using $\mathrm{H}_{2}$ absorber. The $\mathrm{H}_{2}$ absorber enables cooling to smaller beam size with less halo.
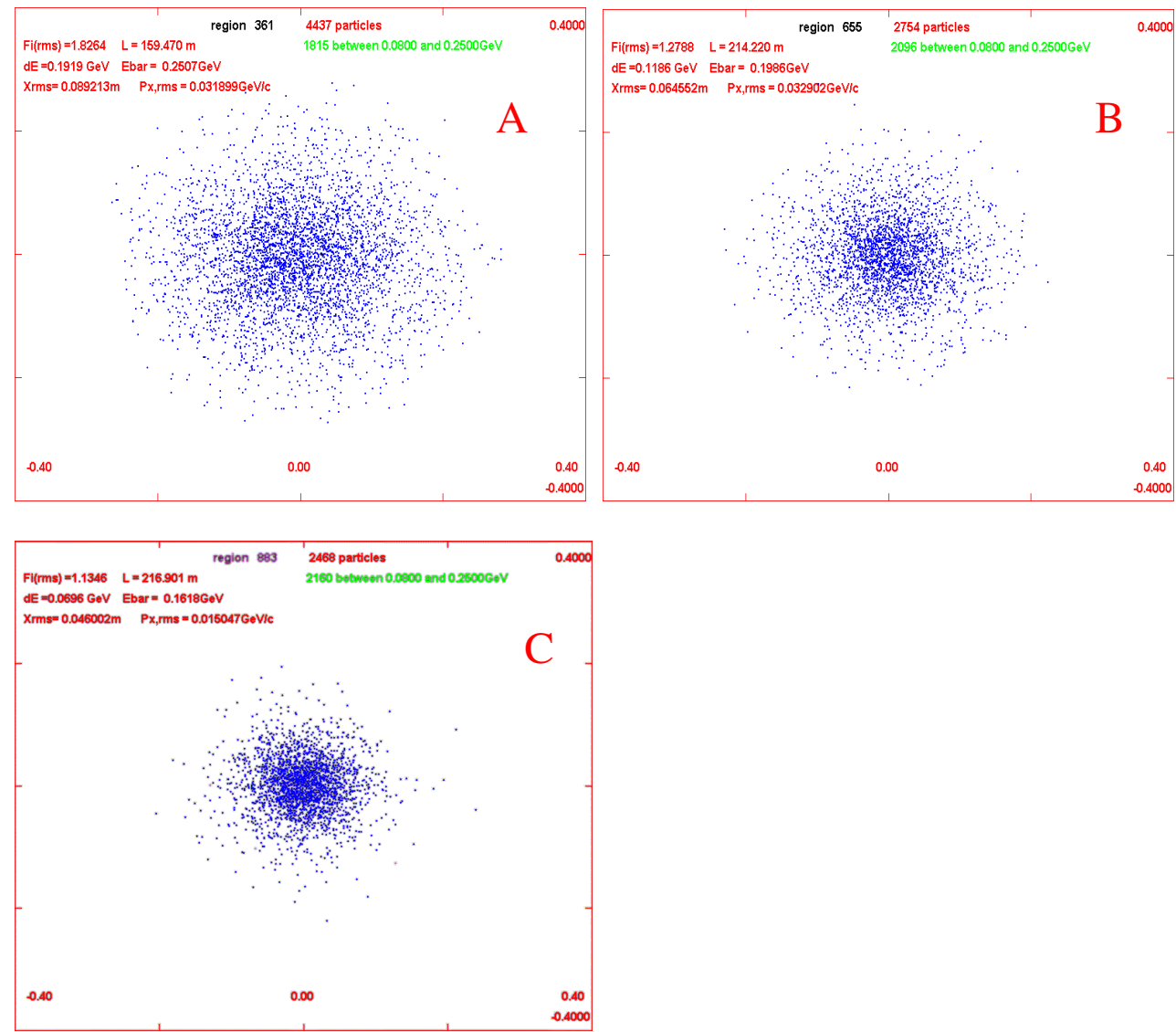